\title{
Maximal symplectic packings in $\mathbb{P}^{2}$
}

\author{
Emmanuel Opshtein
}

\begin{abstract}
In this paper we describe the intersections between the balls of maximal symplectic packings of $\mathbb{P}^{2}$. This analysis shows the existence of singular points for maximal packings of $\mathbb{P}^{2}$ by more than three equal balls. It also yields a construction of a class of very regular examples of maximal packings by five balls.
\end{abstract}

\section{Introduction}

The symplectic packing problem is the question of identifying the conditions on the radii of $k$ balls for being able to pack them symplectically in a given manifold. It was first considered by Gromov as a problem whose answer distinguishes symplectic geometry from the volume-preserving one: the restrictions are sometimes stronger than the volume obstruction alone [Gro85]. For instance, the non-squeezing theorem asserts that no ball of radius bigger than one can be packed into the infinite volume cylinder $B^{2}(1) \times \mathbb{R}^{2 n}$. In the special case of $\mathbb{P}^{2}$, this problem has been completely solved. Apart from the volume obstruction, symplectic packings by less than eight balls are submitted to finitely many purely symplectic obstructions discovered by Gromov [Gro85] and by McDuff and Polterovich [MP94]. Biran proved that the symplectic obstructions disappear for more than nine balls [Bir99].

This paper is aimed at describing what the packings of $\mathbb{P}^{2}$ by balls of maximal radii look like. We are particularly interested in understanding their intersection properties. Let us first define our setting. Throughout this paper, the symplectic structure on $\mathbb{P}^{2}$ is given by the standard FubiniStudy form normalized so that $\int_{\mathbb{C P}^{1}} \omega=\pi$. With this normalization, $\mathbb{C P}^{2} \backslash \mathbb{C P}^{1}$ is symplectomorphic to the standard unit open ball $B^{4}(1)$ in $\mathbb{C}^{2}$.

Definition 1.1. A maximal symplectic packing of $M$ by $k$ balls is a symplectic embedding $\varphi$ : $\left(B\left(r_{1}\right) \amalg \ldots \amalg B\left(r_{k}\right), \omega_{\mathrm{st}}\right) \hookrightarrow(M, \omega)$ where the radii are such that there exists no symplectic packing of $M$ by balls of radii $\left(r_{1}, \ldots, r_{i}+\varepsilon, \ldots, r_{k}\right)$. It will be said to be smooth if each $\varphi_{i}:=\varphi_{\mid B\left(r_{i}\right)}$ extends to a smooth embedding of the closed balls. It will be said to be regular if these maps have only a finite number of singular points on the boundary: each $\varphi_{i}$ extends to a topological embedding of the closed ball which is a smooth embedding of $\overline{B\left(r_{i}\right)} \backslash\left\{p_{i}^{1}, \ldots, p_{i}^{n_{i}}\right\}$.

The space of regular maximal symplectic packings by a fixed number of balls is naturally endowed with the Hausdorff topology for compact sets. Throughout this paper, genericity is meant with respect to this topology, and should be understood in a strong sense: a property is generic if it is true for an open dense set. Our first theorem deals with smooth maximal symplectic packings of $\mathbb{P}^{2}$.

Theorem 1. The generic intersection patterns between the balls of smooth maximal symplectic packings of $\mathbb{P}^{2}$ are as follows.

Received 3 November 2006, accepted in final form 26 March 2007, published online 9 November 2007.

2000 Mathematics Subject Classification 53D05 (primary), 57R17 (secondary).

Keywords: symplectic topology, symplectic packings.

This research was supported by The Israel Science Foundation (Grant No. 1227/06).

This journal is (c) Foundation Compositio Mathematica 2007. 
(a) Generically, the closed balls of a smooth maximal symplectic packing of $\mathbb{P}^{2}$ by two balls intersect precisely along one common Hopf circle of their boundary.

(b) Generically, any two closed balls of a smooth maximal symplectic packing of $\mathbb{P}^{2}$ by three equal balls intersect precisely along one common Hopf circle of their boundary.

(c) Generically, the two smallest balls of a smooth maximal symplectic packing of $\mathbb{P}^{2}$ by three non-equal balls do not intersect, while the intersection of the biggest ball with any of the others is exactly one common Hopf circle of their boundaries.

(d) There exists no smooth maximal symplectic packing of $\mathbb{P}^{2}$ by more than three equal balls.

The Hopf circles of a ball are the characteristic leafs of its boundary (see $\S 3.1$ ). Theorem 1 is better understood in the light of Karshon's examples [MP94]: it says that generic smooth maximal symplectic packings look very much like those she produced (see $\S 2$ ). The idea is to translate the maximality property to the existence of characteristic circles in the intersections of the boundary spheres. These characteristics give rise to (maybe singular) symplectic spheres, whose intersection properties lead to the desired uniqueness. The approach is based on a strong connection observed by Paternain, Polterovich and Siburg [PPS03] or Laudenbach and Sikorav [LS94] between symplectic non-removable intersection and closed characteristics.

The importance of the characteristic foliation in the present study is the reason for our definition of smooth or regular maximal symplectic packing to be so restrictive. Before going further, a discussion of the existence of the objects under consideration is needed. A non-constructive argument due to McDuff shows that there always exist symplectic packings by open balls of maximal radii, with no guaranteed boundary regularity. On the other hand, several explicit examples are available. We already mentioned Karshon's smooth packings by two or three balls. Generalizing her construction, Traynor [Tra95] and Schlenk [Sch05] produced examples of maximal symplectic packings of $\mathbb{P}^{2}$ by five and six balls, which unfortunately fail by far to be regular. This is not surprising in view of Theorem 1(d). As far as we are concerned, the achieved boundary regularity is nevertheless as difficult to handle as McDuff's abstract maximal packings: no convenient notion of characteristic foliation on the boundary of the balls can be defined. The second result of this paper exhibits the relevance of our definition of regularity. Allowing only finitely many singularities enables us to produce interesting maximal packings of $\mathbb{P}^{2}$, at least by five balls.

THEOREM 2. There exist regular maximal symplectic packings of $\mathbb{P}^{2}$ by five equal balls.

The construction relies on a decomposition theorem of Kähler manifolds due to Biran [Bir01].

Our third result generalizes Theorem 1 to the regular setting. The intersections between the balls of a regular maximal symplectic packing is a union of Hopf circles of the boundary spheres which provide a 'grid' of a topological 2-fold, symplectic away from its singularities.

THEOREM 3. Given a regular maximal symplectic packing of a symplectic manifold, there exists at least one 'supporting surface': a closed topological surface covered by the balls of the packing and whose intersection with any ball is a union of smooth symplectic discs bounded by Hopf circles. Generically, the balls of the packing intersect exactly along the Hopf circles contained in the supporting surfaces.

The existence result above can be sharpened when the singularities are simple enough (see Definition 5.3 of packings of simple type). For five balls, for instance, the intersection pattern must be the same as in the constructed examples (Theorem 2).

Theorem 4. Regular maximal symplectic packings of $\mathbb{P}^{2}$ by five equal balls which have simple type have exactly one supporting surface, of symplectic area $2 \pi$, intersecting each ball in exactly 


\section{E. Opshtein}

one Hopf disc. Generically, these maximal packings thus intersect along exactly one Hopf circle of each of the boundary spheres.

The paper is organized as follows. In $\S 2$, we discuss previously known examples of maximal symplectic packings and we construct new ones (Theorem 2). These examples shed some light on Theorems 1, 3 and 4 by providing relevant illustrations. In the third section, we explain the link between non-removable intersections and characteristic foliations in the setting of smooth balls, and prove Theorem 1. The purpose of $\S 4$ is to adapt the tools of $\S 3$ to non-smooth objects. We prove Theorem 3 in $\S 5$ and conclude by a technical paragraph aimed at smoothening the supporting surfaces to prove Theorem 4 .

\section{Examples of maximal symplectic packings}

The aim of this section is to provide examples of smooth or regular symplectic packings. We describe them in the light of the results stated in the Introduction.

\section{Karshon's construction}

Examples of smooth maximal symplectic packings of $\mathbb{P}^{2}$ were given by Karshon [MP94] and Traynor [Tra95]. They can be described in the following way. The momentum map (or the action-angle coordinates) presents $\mathbb{P}^{2}$ as a singular bundle over a closed triangle with two-dimensional tori as generic fibres. The balls forming the packing are fibred by these tori and project by the momentum map to closed triangles (see Figure 1).

The packing is smooth in the sense of Definition 1.1. Indeed, one of the balls is obtained by restricting the symplectic embedding

$$
\begin{aligned}
\Phi: B^{4}(1) & \longrightarrow \mathbb{P}^{2} \\
\left(z_{1}, z_{2}\right) & \longmapsto\left[\sqrt{1-|z|^{2}}: z_{1}: z_{2}\right]
\end{aligned}
$$

to the ball of radius one-half. This ball is obviously a smooth one because it is the restriction of a bigger one. Now the two other balls are obtained from the first one by the global symplectomorphisms of $\mathbb{P}^{2}$ which consist of exchanging the coordinates $\left[z_{0}: z_{1}: z_{2}\right] \mapsto\left[z_{1}: z_{0}: z_{2}\right]$ or $\left[z_{1}: z_{2}: z_{0}\right]$. Thus all three balls are smooth, and the packing itself is a smooth one.

Note that the intersection between the closed balls in these examples is precisely one common circle of the Hopf fibration of the boundary spheres (point $I$ ). Moreover, the fibre over the line $A C$ forms a two-dimensional sphere covered by the union of the two balls and whose intersection with each ball is a disc bounded by a Hopf circle. It is thus precisely one of the 'supporting surfaces' of the maximal packing. Theorem 1 says that every smooth maximal packing of $\mathbb{P}^{2}$ by two or three balls always has the same intersection patterns as those examples.
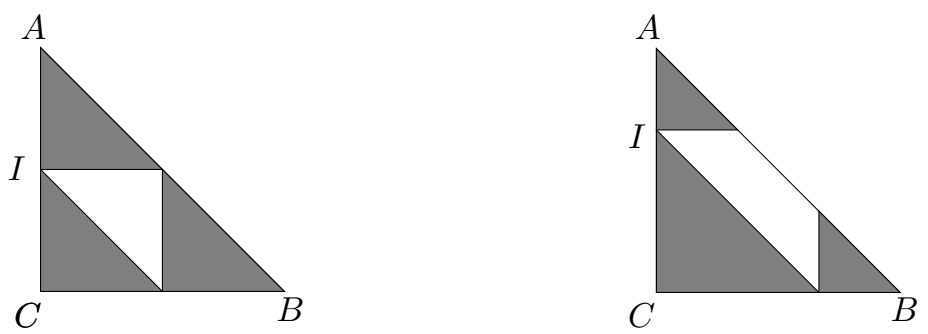

Figure 1. Maximal symplectic packings of $\mathbb{P}^{2}$ by three balls. 
From this example, it is also easy to see that the assumption concerning the equality of the balls in Theorem $1(\mathrm{~d})$ cannot be dropped. Consider actually a symplectic packing $\left\{B_{1}, B_{2}\right\}$ of $\mathbb{P}^{2}$ by smooth balls of radii $r_{2} \ll 1$ and $r_{1}=\sqrt{1-r_{2}^{2}}$, centred at the points $A$ and $C$ with the notation of Figure 1. Looking at them in $B^{4}(1)$ rather than $\mathbb{P}^{2}$ (their interiors do not intersect the $\mathbb{P}^{1}$ at infinity), we see one big ball $B_{1}^{\prime}$ centred at the origin and one thin compact set $B_{2}^{\prime}$ (not a closed ball any more) very close to the Hopf circle of $\partial B^{4}(1)$ corresponding to $A$. There clearly exist approximately $1 / r_{2}^{2}$ unitary transformations of $\mathbb{C}^{2}$ (leaving $B_{1}^{\prime}$ invariant) which take $B_{2}^{\prime}$ to disjoint compact sets. After collapsing the Hopf circles of $\partial B^{4}(1)$ to get $\mathbb{P}^{2}$, these compact sets transform to disjoint symplectic balls of the same radius $r$. Together with $B_{1}$, they provide a smooth maximal symplectic packing of $\mathbb{P}^{2}$ by approximately $1 / r_{2}^{2}$ balls.

\section{Biran's decomposition theorem (see [Bir01])}

The examples of regular packings to come are based on a decomposition result due to Biran which we describe now briefly. Given an integral Kähler manifold $(M, \omega, J)$ (i.e. with $[\omega] \in H^{2}(M, \mathbb{Z})$ ), there always exists a complex hypersurface $X$ which is Poincaré dual to $k \omega$ for some big enough integer $k$. Biran showed that there exists a CW-complex $\Delta_{X} \subset M$ (of empty interior) associated to this hypersurface, whose complement in $M$ is a standard symplectic disc bundle over $X$. It turns out that some maximal packings appear very clearly in these special coordinates. In order to explain this, let us discuss briefly the structure of these standard bundles. Their symplectic type is that of the unit disc bundle associated to a Hermitian line bundle of first Chern class $c_{1}=[k \tau]$, where $\tau=\omega_{\mid X}$. The symplectic structure itself is explicitly given by

$$
\omega:=\pi^{*} \tau+d\left(r^{2} \alpha\right)
$$

where $r$ is the radial coordinate in the fibre and $\alpha$ is the 1-form whose restriction to the fibres is $\alpha_{\mid \pi^{-1}(x)}=1 / k d \theta$ on $E \backslash\{r=0\}$ and $d \alpha=-\pi^{*} \tau$. Notice that, although $\theta$ is not defined globally on $E$ because of non-vanishing Chern class, the differential form ' $d \theta$ ' is perfectly defined away from the zero section.

The connection between standard symplectic bundles and balls comes from the following simple observation. The restriction of these bundles to a symplectic ellipsoid in $X$ is symplectomorphic to an ellipsoid. In the following lemma, $\mathcal{E}_{a}=\mathcal{E}_{\left(a_{1}, \ldots, a_{n}\right)}$ denotes the standard ellipsoid in $\mathbb{C}^{n}$ :

$$
\mathcal{E}_{a}:=\left\{\left(z_{1}, \ldots, z_{n}\right) \in \mathbb{C}^{n} \mid \frac{\left|z_{1}\right|^{2}}{a_{1}}+\cdots+\frac{\left|z_{n}\right|^{2}}{a_{n}}<1\right\} .
$$

Lemma 2.1. Consider the trivial disc bundle $\pi: \mathcal{E}_{a} \times \mathbb{D} \longrightarrow \mathcal{E}_{a}$ over an ellipsoid of $\mathbb{C}^{n}$, equipped with the symplectic structure defined by $\omega:=\pi^{*} \omega_{\text {st }}+d\left(r^{2} \alpha\right), \alpha_{\mid\{x\} \times \mathbb{D}}=1 / k d \theta$ and $d \alpha=-\pi^{*} \omega_{\text {st }}$, where $(r, \theta)$ are the polar coordinates on $\mathbb{D}$. Then there exists a smooth function $h: \mathcal{E}_{a} \longrightarrow \mathbb{R}$ such that the map

$$
\begin{aligned}
\Phi:\left(\mathcal{E}_{a} \times \mathbb{D}, \omega\right) & \longrightarrow\left(\mathcal{E}_{a, 1 / k}, \omega_{\mathrm{st}}\right) \\
(z, w) & \longmapsto\left(\sqrt{1-|w|^{2}} z, \frac{1}{\sqrt{k}} e^{i h(z)} w\right)
\end{aligned}
$$

is a symplectomorphism.

Proof. Consider the coordinates $(z, w)=\left(r_{1}, \theta_{1}, \ldots, r_{n}, \theta_{n}, r, \theta\right)$ on $\mathcal{E}_{a} \times \mathbb{D}$. The symplectic form $\omega$ is given in these coordinates by

$$
\omega=\sum_{i=1}^{n} d r_{i}^{2} \wedge d \theta_{i}+d\left(r^{2} \alpha\right)
$$




\section{E. Opshtein}

Taking into account the identities $\alpha_{\mid\{x\} \times \mathbb{D}}=1 / k d \theta$ and $d \alpha=-\sum d r_{i}^{2} \wedge d \theta_{i}$, we get

$$
\begin{aligned}
\omega & =\left(1-r^{2}\right) \sum_{i=1}^{n} d r_{i}^{2} \wedge d \theta_{i}+d r^{2} \wedge \alpha \\
& =\sum_{i=1}^{n} d\left[\left(1-r^{2}\right) r_{i}^{2}\right] \wedge d \theta_{i}+d r^{2} \wedge\left[\alpha+\sum_{i=1}^{n} r_{i}^{2} d \theta_{i}\right] \\
& =\sum_{i=1}^{n} d\left[\left(1-r^{2}\right) r_{i}^{2}\right] \wedge d \theta_{i}+\frac{1}{k} d r^{2} \wedge[d \theta+\beta],
\end{aligned}
$$

where $\beta:=k\left[\alpha-1 / k d \theta+\sum_{i=1}^{n} r_{i}^{2} d \theta_{i}\right]$. The form $\beta$ is defined on $\mathcal{E}_{a} \times(\mathbb{D} \backslash\{0\})$ and is closed. Moreover, its action on the fundamental group of $\mathcal{E}_{a} \times \mathbb{D} \backslash\{0\}$ is trivial because $\beta_{\mid\{x\} \times \mathbb{D}}=0$. Hence there is a smooth function $h: \mathcal{E}_{a} \times \mathbb{D} \backslash\{0\} \longrightarrow \mathbb{R}$ such that $\beta=d h$. Notice now that $h$ does not depend on $w$ because $\beta$ vanishes on the vertical discs. This function thus extends to $\mathcal{E}_{a} \times \mathbb{D}$ and depends only on $z$. We finally get

$$
\begin{aligned}
\omega & =\sum_{i=1}^{n} d\left[\left(1-r^{2}\right) r_{i}^{2}\right] \wedge d \theta_{i}+\frac{1}{k} d r^{2} \wedge d(\theta+h) \\
& =\Phi^{*}\left[\sum_{i=1}^{n} d r_{i}^{2} \wedge d \theta_{i}+d r^{2} \wedge d \theta\right],
\end{aligned}
$$

where $\Phi$ is the announced map. It clearly sends $\mathcal{E}_{a} \times \mathbb{D}$ to the ellipsoid $\mathcal{E}_{a, 1 / k}$.

Since a complex hypersurface of a Kähler manifold is Kähler, an obvious iteration leads to the following corollary. It seems to hold true also in general compact symplectic manifolds due to Donaldson's results on the existence of symplectic hypersurfaces [Don96].

Corollary 2.2. Every Kähler manifold has a full packing by one ellipsoid.

\section{Maximal full packing of $\mathbb{P}^{n}$ by $k^{n}$ balls}

We make a digression at this point to explain how to construct a full symplectic packing of $\mathbb{P}^{2}$ by four open balls of radius $1 / \sqrt{2}$. The generalization to $k^{n}$ balls of maximal radius $1 / \sqrt[n]{k}$ in $\mathbb{P}^{n}$ is straightforward. As far as I know, although their existence is well known from McDuff and Polterovich's [MP94] or Traynor's work [Tra95] via McDuff's argument [McD98], no example of such packings has been available up to now.

Consider the quadric $Q:=\left\{z_{0}^{2}+z_{1}^{2}+z_{2}^{2}=0\right\}$ in $\mathbb{P}^{2}$ with homogeneous coordinates $\left[z_{0}: z_{1}: z_{2}\right]$ - recall that it is diffeomorphic to a 2-sphere. Then $\pi: \mathbb{P}^{2} \backslash \mathbb{R} \mathbb{P}^{2} \longrightarrow Q$ is a standard disc bundle with fibres of area $\pi / 2$. Choose four disjoint open discs $D_{i}$ in $Q$ of area $\pi / 2, i=1, \ldots, 4$. Then the sets $B_{i}:=\pi^{-1}\left(D_{i}\right) \subset \mathbb{P}^{2}$ are obviously disjoint. Moreover, the previous lemma shows that they are symplectic balls of radius $1 / \sqrt{2}$. Notice also that the boundary singularities of these balls (inavoidable by Theorem $1(\mathrm{~d})$ ) are easily describable in terms of the singularities of the discs $D_{i}$ thanks to the explicit formula for the symplectomorphism $\Phi$ of Lemma 2.1.

\section{Regular maximal packing of $\mathbb{P}^{2}$ by five balls (see Figure 2)}

As above, consider the quadric $Q:=\left\{z_{0}^{2}+z_{1}^{2}+z_{2}^{2}=0\right\}$, and the projection $\pi: \mathbb{P}^{2} \backslash \mathbb{R} \mathbb{P}^{2} \longrightarrow Q$ which gives $\mathbb{P}^{2} \backslash \mathbb{R} \mathbb{P}^{2}$ the structure of a standard disc bundle with fibres of area $\pi / 2$. Cover $Q$ by five closed discs of area $2 \pi / 5$ with finite number of singularities on their boundaries. We claim that we can find the desired balls of the packing inside the sets fibred upon these discs. To see this, we construct a regular symplectic embedding of a ball of radius $R$ into $\pi^{-1}(D)$, where $D$ is any closed disc in $Q$ of area $\pi R^{2}<\pi / 2$, with a finite number of singularities $p_{1}, \ldots, p_{k} \in \partial D$. First identify $\pi^{-1}(D)$ 


\section{MaXimal SYMPLECTIC PACKINGS IN $\mathbb{P}^{2}$}

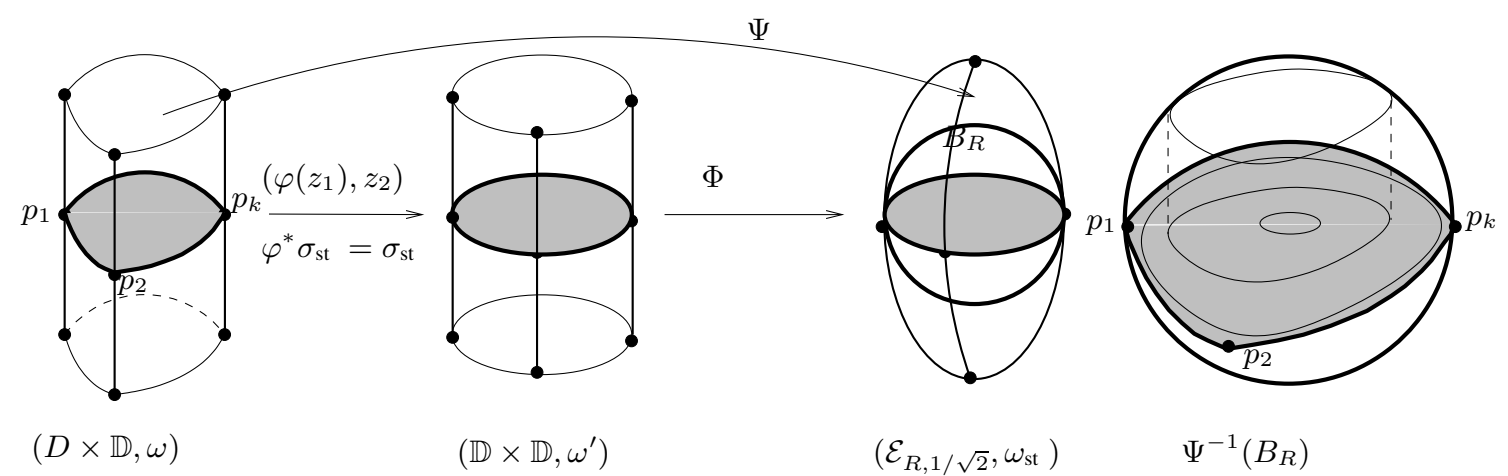

FiguRE 2. Regular packing of a standard disc bundle over a disc by a ball.

with $D \times \mathbb{D}$. Then $\left(\pi^{-1}(D), \omega_{F S}\right)$ is symplectomorphic to a standard ellipsoid $\mathcal{E}:=\mathcal{E}_{R, 1 / \sqrt{2}}$ via a map $\Psi$ which is the composition of a fibred map $\varphi \times$ Id sending $D \times \mathbb{D}$ to the standard bidisc $\mathbb{D}_{R} \times \mathbb{D}$ with the map $\Phi$ of Lemma 2.1 where now $k=2$. The boundary regularity of the symplectomorphism $\Psi: \pi^{-1}(D) \longrightarrow \mathcal{E}$ can be described as follows. First it extends to a homeomorphism between $\bar{D} \times \mathbb{D}$ (i.e. $\overline{\pi^{-1}(D)}$ minus the section at infinity) and $\overline{\mathcal{E}} \backslash C_{\infty}$ where $C_{\infty}:=\left\{\left|z_{2}\right|^{2}=1 / \sqrt{2}, z_{1}=0\right\}$. Moreover, this extension is a local diffeomorphism except at the singular points $\bigcup\left\{p_{i}\right\} \times \mathbb{D}$ of $\pi^{-1}(D)$. Now the ellipsoid $\overline{\mathcal{E}}$ contains a Euclidean closed ball $B$ of radius $R$ whose boundary intersects $\partial \mathcal{E}$ only along the 'zero section' $\left\{z_{2}=0,\left|z_{1}\right|=R\right\}$. The map $\Psi^{-1}: B \longrightarrow \pi^{-1}(D)$ is therefore a regular symplectic embedding of a ball of radius $R$ in $\pi^{-1}(D)$.

\section{Non-removable intersections in smooth maximal packings}

The aim of this section is to introduce the main tool of the paper - namely the link between nonremovable intersections in symplectic geometry and characteristic foliations - following [PPS03, LS94]. We also prove Theorem 1. Dealing only with smooth objects first permits us to avoid the technical difficulties arising in the context of regular packings.

Let us explain first how the genericity is achieved in Theorem 1. We define a Hamiltonian perturbation of a maximal packing $\left\{\varphi_{i}\right\}_{i=1, \ldots, k}$ as a family $\left\{\varphi_{i}^{t}\right\}:=\left\{\Phi_{X_{H_{i}}}^{t} \circ \varphi_{i}\right\}$, where the timedependent Hamiltonian functions $H_{i}$ on $M$ are such that the open balls $\varphi_{i}^{t}\left(B\left(r_{i}\right)\right)$ remain disjoint for all $t \in[0, \varepsilon]$. Note that these Hamiltonian perturbations of packings enable us to break intersections. The properties stated in Theorem 1 are generic precisely because they are always true after a possible Hamiltonian perturbation of the packing.

\subsection{Pushing into a ball by a Hamiltonian: Sullivan's lemma}

As explained in [PPS03] and [LS94], the characteristic foliation plays a central role in the phenomena of 'symplectic non-removable intersection'. Loosely speaking, the reason for a compact set inside the smooth boundary of an open set $U$ not to be displaceable inside $\bar{U}$ by a Hamiltonian vector field is that it contains a closed invariant set of the characteristic foliation of $\partial U$.

Definition 3.1. The characteristic distribution $\mathcal{L}$ of a hypersurface $S$ in a symplectic manifold $(M, \omega)$ is the kernel of the restriction of $\omega$ to $S$ (i.e. for all $\left.x \in S, \mathcal{L}_{x}:=\operatorname{ker} \omega_{x \mid T_{x} S}\right)$. The characteristic foliation of $S$ is the integral foliation associated to this one-dimensional characteristic distribution.

The characteristic foliation is obviously preserved by any map $\varphi: S \subset(M, \omega) \longrightarrow S^{\prime} \subset\left(M^{\prime}, \omega^{\prime}\right)$ with $\varphi^{*} \omega_{\mid T S^{\prime}}^{\prime}=\omega_{\mid T S}$. 


\section{E. Opshtein}

Example. On the Euclidean sphere $S(r) \subset \mathbb{C}^{n}$, the characteristic distribution is given by $\mathcal{L}_{x}=$ $\operatorname{Span}_{\mathbb{R}}(i \vec{N}(x))$ where $\vec{N}(x):=x /\|x\|$. The characteristic foliation is thus the classical foliation of $S(r)$ by Hopf circles. Any symplectic smooth (or regular) embedding of a Euclidean closed ball of $\mathbb{C}^{n}$ into $M$ is called a smooth (or regular) symplectic closed ball of $(M, \omega)$. If $B$ is a symplectic closed ball in $M$ corresponding to a regular embedding $\varphi: \overline{B(r)} \longrightarrow M$, the characteristic leaves of $\partial B=\varphi(S(r))$ are the images by $\varphi$ of the Hopf circles of $S(r)$ and will be called the Hopf circles of $B$. Finally, the Hopf discs of a symplectic ball $B$ are the images of the intersections of $B(r)$ with the complex lines of $\mathbb{C}^{n}$.

The vector field $i \vec{N}(x)$ defines and orients the characteristic foliation of the Euclidean sphere $S$ in $\mathbb{C}^{n}$. The Hamiltonian vector field $\vec{X}_{H}$ associated to a smooth function $H: \mathbb{C}^{n} \longrightarrow \mathbb{R}$ points inside $B$ at a point $x$ of $S$ if and only if $i \vec{N} \cdot H(x)<0$. Indeed, we have

$$
\vec{X}_{H} \cdot \vec{N}(x)<0 \quad \Longleftrightarrow \omega\left(\vec{X}_{H}(x), i \vec{N}(x)\right)<0 \quad \Longleftrightarrow \quad d H_{x}(i \vec{N}(x))<0 .
$$

In other words, the Hamiltonian vector field $\vec{X}_{H}$ pushes into $B$ at a point $x$ of $S$ if $H$ is decreasing along the characteristic foliation at this point. The following lemma explains along which compact sets of $S$ one can push into $B$ in a Hamiltonian way. It is a particular case of a more general result due to Sullivan [Sul76] (see also [LS94, LM95]).

Lemma 3.2. Let $K$ be a compact set of $S^{2 n-1} \subset \mathbb{C}^{n}$ and $\mathcal{C}$ the (compact) set of Hopf circles belonging to $K$. There exists a smooth function $H: S^{2 n-1} \longrightarrow \mathbb{R}$ such that $i \vec{N} \cdot H<0$ on $K \backslash \mathcal{C}$ and $d H=0$ on $\mathcal{C}$.

Proof. We first reduce Lemma 3.2 to finding convenient functions on solid tori by a partition of unity argument. Denote the Hopf projection by $\pi: S^{2 n-1} \longrightarrow \mathbb{P}^{n-1}$. Given a locally finite open cover by balls $\mathcal{U}:=\left\{U_{\alpha}\right\}$ of $\pi(K \backslash \mathcal{C})$, the open set $U:=\mathbb{P}^{n-1} \backslash K$ completes $\mathcal{U}$ to a locally finite cover of $\mathbb{P}^{n-1} \backslash \pi(\mathcal{C})$. Consider a smooth partition of unity $\left\{\Phi_{\alpha}, \Phi\right\}$ associated to $\left(U_{\alpha}, U\right)$. Also choose a smooth non-negative function $\theta$ on $\mathbb{P}^{n-1}$ which vanishes on $\pi(\mathcal{C})$ at order two and is positive away from $\pi(\mathcal{C})$. We claim that if we can find functions $h_{\alpha}: \pi^{-1}\left(U_{\alpha}\right) \longrightarrow \mathbb{R}$ such that $i \vec{N} \cdot h_{\alpha}<0$ on $K \backslash \mathcal{C} \cap \pi^{-1}\left(U_{\alpha}\right)$ then the function $H=\theta \circ \pi \sum \lambda_{\alpha} \Phi_{\alpha} \circ \pi \cdot h_{\alpha}$ is of the required type, provided that the positive constants $\lambda_{\alpha}$ are small enough to guarantee the smoothness of $H$ (the choice $\lambda_{\alpha}:=2^{-\alpha}\left\|\Phi_{\alpha} \circ \pi \cdot h_{\alpha}\right\|_{\mathcal{C}^{1}}^{-1}$ is convenient). Indeed, we have

$$
i \vec{N} \cdot H=\theta \circ \pi \sum \lambda_{\alpha} \Phi_{\alpha} \circ \pi \cdot i \vec{N} \cdot h_{\alpha}<0 \quad \text { on } K \backslash \mathcal{C},
$$

and

$$
d H=\left(\sum \lambda_{\alpha} \Phi_{\alpha} \circ \pi \cdot h_{\alpha}\right) d(\theta \circ \pi)+(\theta \circ \pi) d\left(\sum \lambda_{\alpha} \Phi_{\alpha} \circ \pi \cdot h_{\alpha}\right)=0 \quad \text { on } \mathcal{C} .
$$

The first equality holds because $\theta \circ \pi$ and $\Phi_{\alpha} \circ \pi$ are constant along $i \vec{N}$. The second holds because both $\theta \circ \pi$ and $d \theta \circ \pi$ vanish on $\mathcal{C}$.

Thus it only remains to build the cover of $\pi(K \backslash \mathcal{C}) \subset \mathbb{P}^{n-1}$ by balls and the suitable functions on the solid tori which project to these balls by $\pi$. In a neighbourhood of $x \in \pi(K \backslash \mathcal{C})$, the compactness of $K$ allows us to find a local section $s: B_{\delta(x)} \longrightarrow S^{2 n-1}$ of $\pi$ and constants $\varepsilon(x)>0$ such that the intervals

$$
I(\widetilde{x}):=\left\{\Phi_{i \vec{N}}^{t}(s(\widetilde{x})), t \in\right]-\varepsilon(x), \varepsilon(x)[\}, \quad \widetilde{x} \in B_{\delta(x)}
$$

contain no points of $K$. These balls $B_{\delta(x)}$ provide a cover of $\pi(K \backslash \mathcal{C})$, for which we now construct the functions $h_{x}$. For this, first identify $\pi^{-1}\left(B_{\delta(x)}\right)$ with $B_{\delta(x)} \times S^{1}$ by the map $\varphi: B_{\delta(x)} \times S^{1} \longrightarrow$ $\pi^{-1}\left(B_{\delta(x)}\right)$ defined by $\varphi(\widetilde{x}, t):=\Phi_{i \vec{N}}^{t+\varepsilon(x)}(s(\widetilde{x}))$. By $\varphi^{-1}, I(\widetilde{x})$ is taken to $]-2 \varepsilon(x), 0[$ and $i \vec{N}$ to $\partial / \partial t$. 
In these coordinates, we can define the function $h_{x}$ on $B_{\delta(x)} \times S^{1}$ by

$$
\left\{\begin{array}{l}
h_{x}(\widetilde{x}, t)=-t \text { for } t \in[0,1-2 \varepsilon(x)] \\
h_{x} \text { is smooth on } B_{\delta(x)} \times S^{1}
\end{array}\right.
$$

Such a function can obviously be defined, and it fits with the requirement $i \vec{N} \cdot h_{\alpha}<0$ on $K \backslash \mathcal{C} \cap$ $\pi^{-1}\left(U_{\alpha}\right)$.

Corollary 3.3 (Perturbation procedure for smooth balls). Let $M$ be a symplectic manifold, $B \subset$ $M$ a smooth symplectic closed ball and $U$ an open set of $M$. Assume that $U \cap B=\emptyset$, denote $K:=\partial U \cap B$ and $\mathcal{C}$ the Hopf circles of $\partial B$ contained in $K$. Then there is a Hamiltonian function $H$ on $M$ such that $\Phi_{X_{H}}^{\varepsilon}(B) \cap \bar{U}=\Phi_{X_{H}}^{\varepsilon}(B) \cap \partial U=\mathcal{C}$ for all small enough $\varepsilon>0$.

Proof. It is well known that there exists a symplectomorphism $\Phi$ between a neighbourhood $N(\partial B)$ of $\partial B$ in $M$ and a neighbourhood $N(S)$ of $S(r)$ in $\mathbb{C}^{n}$. This map sends $K$ to $K^{\prime}=\Phi(K)$, and $\mathcal{C}$ to $\mathcal{C}^{\prime}=\Phi(\mathcal{C})$, where $\mathcal{C}^{\prime}$ is exactly the set of Hopf circles of $S(r)$ contained in $K^{\prime}$. Consider a function $h$ on $S(r)$ associated to $\left(K^{\prime}, \mathcal{C}^{\prime}\right)$ as in Lemma 3.2. Extend it to a smooth function of $\mathbb{C}^{n}$ with compact support in $N(S)$. The function $H:=h \circ \Phi$, a priori defined on $N(\partial B)$, can be extended to $M$ by setting $H=0$ outside $N(\partial B)$. As explained above, the vector field $X_{H}$ points inside $B$ on $K \backslash \mathcal{C}$ and vanishes on $\mathcal{C}$.

This corollary is unfortunately only useful when dealing with smooth balls. In our context, $B$ will usually be one of the (only regular) balls $B_{i}$ and $U$ the union of the other balls $\bigcup_{j \neq i} B_{j}$. We will prove the counterpart of this perturbation procedure for regular symplectic closed balls in the next section (see Proposition 4.3).

\subsection{Symplectic spheres in maximal packings}

We show here that any Hopf circle in the intersection of two symplectic balls along their boundaries gives rise to its own supporting sphere.

LEMma 3.4. Let $B_{1}, B_{2}$ be regular symplectic closed balls of a symplectic manifold $(M, \omega)$ with disjoint interior, of radii $r_{1}, r_{2}$ and centres $O_{1}, O_{2}$. Given any common Hopf circle $C$ of $\partial B_{1}$ and $\partial B_{2}$, one can construct a topological 2-sphere $S_{C}$ of $M$ passing through $C, O_{1}, O_{2}$, smooth except along $C$ and with symplectic area $\pi\left(r_{1}^{2}+r_{2}^{2}\right)$. In dimension four, these spheres intersect precisely in $O_{1}$ and $O_{2}$ with intersection number 1 at each point.

Proof. Let $\varphi_{i}: \overline{B\left(r_{i}\right)} \longrightarrow B_{i}$ be the corresponding symplectic embeddings and $C_{i}:=\varphi_{i}^{-1}(C)$. Then $C_{i}$ is a Hopf circle of $\partial B\left(r_{i}\right)$ and bounds a holomorphic disc $\mathbb{D}_{C_{i}} \subset B\left(r_{i}\right)$. Its image by $\varphi_{i}$ is a symplectic disc $D_{i}$ in $B_{i}$ of area $\pi r_{i}^{2}$ passing through $O_{i}$ and bounded by $C$ (a Hopf disc). Since the interiors of $B_{1}, B_{2}$ are disjoint, when gluing $D_{1}$ with $D_{2}$ along their common boundary we get a topological 2-sphere $S_{C}$ which is smooth except on $C$ and has area $\mathcal{A}\left(S_{C}\right)=\pi r_{1}^{2}+\pi r_{2}^{2}$. By construction, two such spheres $S_{C}, S_{C^{\prime}}$ intersect only at $O_{1}, O_{2}$, and in dimension four their intersection numbers at $O_{i}$ is 1 because they are the same as the intersection number of $\mathbb{D}_{C_{i}}, \mathbb{D}_{C_{i}^{\prime}}$ in $B^{4}\left(r_{i}\right)$.

\subsection{Smooth maximal packings: proof of Theorem 1}

Proof of Theorem 1(a). Fix a smooth maximal symplectic packing of $\mathbb{P}^{2}$ by two balls $\left\{B_{1}, B_{2}\right\}$ of radii $r_{1}, r_{2}$ verifying the maximality condition $r_{1}^{2}+r_{2}^{2}=1$. Set $K:=\partial B_{1} \cap \partial B_{2}$ and let $\mathcal{C}$ be the set of Hopf circles of $\partial B_{1}$ contained in $K$. Note that such a circle is also a Hopf circle of $\partial B_{2}$ because $\partial B_{1}$ and $\partial B_{2}$ are tangent along $K$. The perturbation procedure of Corollary 3.3 shows that, after a possible Hamiltonian perturbation of the packing, we can assume that $K=\mathcal{C}$. 


\section{E. Opshtein}

Gromov's work [Gro85] shows that the balls of a smooth maximal packing of $\mathbb{P}^{2}$ by two balls cannot be disjoint (we also prove this in Proposition 4.1 in the more general setting of regular mappings). This implies that no Hamiltonian perturbation of our packing can lead to disjoint balls. In view of the perturbation procedure of Corollary 3.3, $\mathrm{K}$ must contain at least one Hopf circle of $\partial B_{1}$, so $\mathcal{C}$ is not empty. To prove that $\mathcal{C}$ is exactly one Hopf circle, we argue by contradiction and assume that $\mathcal{C}$ contains two circles $C$ and $C^{\prime}$. The topological 2-spheres $S_{C}$ and $S_{C^{\prime}}$ given by Lemma 3.4 intersect precisely at $O_{1}, O_{2}$ with intersection numbers 1 at each point. On the other hand, they both have symplectic area $\pi\left(r_{1}^{2}+r_{2}^{2}\right)=\pi$ so they are in the homology class of a line $L$ in $\mathbb{P}^{2}$. We thus have $2=S_{C_{1}} \cdot S_{C_{2}}=L \cdot L=1$, which is a contradiction.

Proof of Theorem 1(b,c). Let $B_{1}, B_{2}, B_{3}$ be the closed balls of our smooth maximal packing. We can assume that their radii satisfy $r_{1} \geqslant r_{2} \geqslant r_{3}$. Then the maximality condition is [Gro85]

$$
r_{1}^{2}+r_{2}^{2}=r_{1}^{2}+r_{3}^{2}=1 \text {. }
$$

As above, set $K_{i j}:=B_{i} \cap B_{j}=\partial B_{i} \cap \partial B_{j}$ and let $\mathcal{C}_{i j}$ be the set consisting of the Hopf circles of $K_{i j}$. The first step is to prove that we can get rid of $K_{i j} \backslash \mathcal{C}_{i j}$ by a Hamiltonian perturbation. Unlike in the two-balls situation, this is not obvious at first glance because a Hopf circle of $\partial B_{1}$ could a priori be covered by $\partial B_{2} \cup \partial B_{3}$ without being in any $K_{i j}$. But notice that the $K_{i j}$ are pairwise disjoint compact sets (and remain so after perturbation) because the intersection of two of the $K_{i j}$ is precisely $B_{1} \cap B_{2} \cap B_{3}$. This intersection must be empty because any point of it would be a singular point of at least one of the balls. Since Hopf circles are connected, we deduce that $K_{12} \cup K_{13}$ contains no Hopf circles besides those in $\mathcal{C}_{12}$ and $\mathcal{C}_{13}$. After a Hamiltonian perturbation of $B_{1}$ according to Corollary 3.3, we can assume that $K_{1 i}=\mathcal{C}_{1 i}$. After a second Hamiltonian perturbation, this time of $B_{2}$, we can also assume that $K_{23}=\mathcal{C}_{23}$.

Case 1: Theorem 1(b) $\left(r_{1}=r_{2}=r_{3}=1 / \sqrt{2}\right)$. In this case, any two of the balls $B_{1}, B_{2}, B_{3}$ form a maximal symplectic packing of $\mathbb{P}^{2}$ by two balls. As such, and in view of Theorem $1(\mathrm{a}), K_{i j}=\mathcal{C}_{i j}$ contains exactly one circle.

Case 2: Theorem 1(c) $\left(r_{1}>r_{2}=r_{3}\right)$. In this case, $\left(B_{1}, B_{2}\right)$ and $\left(B_{1}, B_{3}\right)$ form maximal symplectic packings of $\mathbb{P}^{2}$ by two balls. As in the proof of Theorem $1(\mathrm{a})$, we conclude that $\mathcal{C}_{12}$ and $\mathcal{C}_{13}$ contain exactly one circle. Finally, if $\mathcal{C}_{23}$ would contain a circle, Lemma 3.4 would associate to it a toplogical 2-sphere of symplectic area $\left.\pi\left(r_{2}^{2}+r_{3}^{2}\right) \in\right] 0, \pi\left[\right.$, which is impossible, so $\mathcal{C}_{23}$ is empty.

Remark 3.5. This last argument shows that any two closed symplectic balls of radii $r_{1}, r_{2}$ in $\mathbb{P}^{2}$ with disjoint interiors can intersect along a full Hopf circle of the boundary of one of them (therefore of both) only if $r_{1}^{2}+r_{2}^{2} \in \mathbb{N}$.

Proof of Theorem 1(d). Suppose by contradiction that $n$ closed balls $B_{1}, \ldots, B_{n}$ of the same radius $r$ constitute a maximal packing of $\mathbb{P}^{2}(n \geqslant 4)$.

We know from [MP94] and [Bir99] that, for $n=4$ and $n \geqslant 9$, such a packing fills the space. Since $B_{1} \cup B_{2}$ does not fill the whole of $\mathbb{P}^{2}$, there exists a point $p \in \overline{\partial B_{1} \cap \partial B_{i}} \backslash \operatorname{Int}_{\partial B_{1}} \partial B_{1} \cap \partial B_{i}$ of the boundary of $\partial B_{1} \cap \partial B_{i}$ in $\partial B_{1}$. Such a point belongs to a third ball $B_{j}$ so our packing by $B_{1}, \ldots, B_{n}$ is not smooth, as already noted in the last paragraph. Moreover, the radius of the balls is not greater than $\sqrt{2 / 5}<\sqrt{1 / 2}$ for $n=5,6,7,8$. As before, we are in a non-removable intersection situation: the ball $B_{1}$ cannot be disjoint from the union of the other ones $\bigcup_{j \neq 1} B_{j}$ (see Proposition 4.1). In view of Corollary 3.3, this means that there is a Hopf circle $C$ of $\partial B_{1}$ which is also covered by $\bigcup_{j \neq 1} \partial B_{j}$. Assume for instance that $C \cap \partial B_{2} \neq \emptyset$. Since $r_{1}^{2}+r_{2}^{2} \leqslant 4 / 5<1$, Remark 3.5 shows that $C$ cannot be entirely contained in $\partial B_{2}$. So there is a point $p$ in the boundary of $\partial B_{2}$ in $C$. Such a point also belongs to another ball, so it is an intersection point of $\partial B_{1}, \partial B_{2}$ and $\partial B_{i}$ for $i \neq 1,2$. Our packing cannot be smooth. 


\subsection{A digression on Gromov's two-packing theorem}

We end this section with a remark. The previous analysis on non-removable intersections provides an interesting interpretation of Gromov's theorem on two-packings. Consider a packing of $\mathbb{P}^{2}$ by two smooth balls $\left\{B_{1}, B_{2}\right\}$ of radii $r_{1}, r_{2}$. Assume that we know the following assertion to hold true.

Every non-maximal two packing of $\mathbb{P}^{2}$ extends to a smooth maximal packing.

Then from the previous analysis, we conclude that the maximal extensions $\left\{B_{1}^{\prime}, B_{2}^{\prime}\right\}$ of $\left\{B_{1}, B_{2}\right\}$ intersect along a common Hopf circle of their boundaries, so their radii verify $r_{1}^{\prime 2}+r_{2}^{\prime 2} \in \mathbb{N}$ by Remark 3.5. Taking into consideration the volume restriction $r_{1}^{\prime 4}+r_{2}^{\prime 4} \leqslant 1$, we immediately get that $r_{1}^{\prime 2}+r_{2}^{\prime 2}=1$, so $r_{1}^{2}+r_{2}^{2} \leqslant 1$. In this approach, the whole difficulty of the two-packing theorem thus lies on the assertion $(*)$. This assertion is a posteriori true, when knowing the two-packing theorem, Karshon's examples and McDuff's result on the connectedness of the space of symplectic packings in $\mathbb{P}^{2}$.

\section{Regularizations and extensions of regular embeddings}

This purely technical section is aimed at extending the perturbation procedure described in Corollary 3.3 to non-smooth balls. We first explain how to extend slightly a symplectic open ball in an open manifold $M$. It should be noticed that the following proposition applies to regular embeddings of a ball.

Proposition 4.1. Let $\varphi: B(r) \hookrightarrow M$ be a symplectic embedding of a Euclidean ball. Assume that $\varphi$ extends smoothly to an embedding of $\overline{B(r)} \backslash K$ into $M$ where $K$ is a closed subset of $\partial B(r)$. If $K$ contains no Hopf circle of $\partial B(r)$ then, for any open neighbourhood $V$ of $\overline{\operatorname{Im} \varphi}$, there is a symplectic embedding $\widetilde{\varphi}_{\varepsilon}: B(r+\varepsilon) \hookrightarrow V$ for $\varepsilon$ small enough.

If $K=\emptyset$ then $\widetilde{\varphi}_{\varepsilon}$ can be chosen to be an extension of $\varphi$, i.e., $\widetilde{\varphi}_{\varepsilon \mid B(r)}=\varphi$.

The non-squeezing theorem shows that this proposition is sharp in the sense that $K$ indeed has to be assumed not to contain any Hopf circle.

Proof. When the singular locus is empty $(K=\emptyset)$, this proposition is very classical. We nevertheless give its brief proof since it serves as a basis for the non-smooth case. In this situation, the image $\varphi(\partial B(r))$ is a smooth hypersurface of $M$. With $S:=\partial B(r)$, the regular neighbourhood theorem gives a symplectomorphism $\psi: N_{\varepsilon}(S) \longrightarrow N(\varphi(S))$ from an $\varepsilon$-neighbourhood of the standard sphere of radius $r$ in $\mathbb{C}^{n}$ to a neighbourhood of $\varphi(S)$ in $M$ (see [Wei71] or [MS98, p. 101]). Moreover, this map can be chosen in such a way that $\psi_{\mid S}=\varphi_{\mid S}$ and $\psi^{\prime}(x) \vec{N}(x)=\varphi^{\prime}(x) \vec{N}(x)$ for any point $x \in S$ $(\vec{N}(x)$ again denotes $x /\|x\|)$. The map $\varphi_{\varepsilon}: B(r+\varepsilon) \longrightarrow M$ defined by

$$
\varphi_{\varepsilon}(x):= \begin{cases}\varphi(x) & \text { for } x \in B, \\ \psi(x) & \text { for } x \in N_{\varepsilon}(S) \backslash B\end{cases}
$$

is $\mathcal{C}^{1}$-smooth, and hence a symplectic embedding of the ball of radius $r+\varepsilon$ into $M$.

For singular hypersurfaces, the standard neighbourhood theorem is not valid any more, hence the above proof has no straightforward generalization. However, it can be easily adapted by using a regularization trick explained in Lemma 4.2 below. It states that under the extension condition on $\varphi$ in Proposition 4.1, there exists a 'regularization' $\widetilde{\varphi}: \overline{B(r)} \hookrightarrow M$ which is a symplectic embedding of the closed ball into the prescribed neighbourhood $V$ of $\varphi(\overline{B(r)} \backslash K)$. Now the extension $\widetilde{\varphi}_{\varepsilon}$ of $\widetilde{\varphi}$ constructed in the case $K=\emptyset$ gives the desired map.

The result we have used in this proof is a particular case of the following lemma for $\mathcal{C}=\emptyset$. This more general version will nevertheless be useful in order to generalize Corollary 3.3. 


\section{E. Opshtein}

Lemma 4.2. Let $\varphi: \overline{B(r)} \backslash K \hookrightarrow M$ be a symplectic embedding of a closed ball minus a singular compact set $K \subset \partial B(r)$ inside an open manifold $M$. Denote by $\mathcal{C}$ the set of Hopf circles of $\partial B(r)$ contained in $K$. There exists a symplectic embedding $\widetilde{\varphi}$ of $\overline{B(r)} \backslash \mathcal{C}$ into any given neighbourhood $V$ of $\operatorname{Im} \varphi=\varphi(\overline{B(r)} \backslash K)$.

Proof. Consider an open set $U \subset \partial B(r) \backslash K$ which contains at least one point of each Hopf circle of $\partial B(r) \backslash \mathcal{C}$. As in the previous proof, $\varphi$ can be extended to a symplectic embedding of a shell $U_{\varepsilon}:=\{[x,(1+\varepsilon(x)) x[, \quad x \in U\}$, where $\varepsilon$ is a small positive function on $U$. For $\varepsilon$ sufficiently small, $\varphi$ sends $B_{\varepsilon}^{\prime}:=B(r) \backslash K \cup U_{\varepsilon}$ into $V$. Lemma 3.2 shows that there exists a smooth function $h: \partial B(r) \longrightarrow \mathbb{R}$ such that $i \vec{N} \cdot h<0$ on $\partial B(r) \backslash(U \cup \mathcal{C})$ with $d h=0$ on $\mathcal{C}$. When extending this function to $\mathbb{C}^{n}$, we get a Hamiltonian function whose flow has the property that, for $t$ small enough, the set $\Phi_{\vec{X}_{h}}^{t}(\overline{B(r)} \backslash \mathcal{C})$ is contained in $B_{\varepsilon}^{\prime}$. The map

$$
\widetilde{\varphi}:=\varphi \circ \Phi_{\vec{X}_{h}}^{t}: \overline{B(r)} \backslash \mathcal{C} \hookrightarrow V
$$

gives the desired symplectic embedding of $\overline{B(r)} \backslash \mathcal{C}$ into $V$.

Before stating the announced generalization of the perturbation procedure of Corollary 3.3 to regular balls, we need to broaden slightly the notion of Hamiltonian perturbation. We say that a path $\left\{\varphi_{t}, B_{t}\right\}$ of regular symplectic balls in $M$ is a regular Hamiltonian deformation if there exist smooth functions $H_{t}$ defined on the interior of $B_{t}$ such that $\varphi_{t}=\Phi_{X_{H_{t}}}^{t} \circ \varphi$. The point is that we do not impose these functions to be defined in all of $M$, so they may have singularities at the boundary. However, we demand the whole path to be made of regular symplectic balls. Now, a regular Hamiltonian perturbation of a packing is a regular Hamiltonian perturbation of each of its balls such that the configuration of the balls at any time $t$ remains a packing. To illustrate this definition, consider the special case of Lemma 4.2 when $M$ is an open manifold with boundary $\partial M$ and $\varphi:(B(r), K) \hookrightarrow(M, \partial M)$ is a regular symplectic ball with a part $K$ of its boundary sent into $\partial M$. Then the map $\widetilde{\varphi}$ is in fact a regular Hamiltonian deformation of $\varphi$ associated to the function $h \circ \varphi^{-1}$. Moreover, $\widetilde{\varphi}_{\mid \mathcal{C}}=\varphi_{\mid \mathcal{C}}$. This obvious remark is exactly the content of the next proposition.

Proposition 4.3 (Perturbation procedure for regular balls). Let $U$ be an open set of a symplectic manifold $M$ and $B$ a regular symplectic ball in $M \backslash U$. Set $K:=\partial B \backslash \partial U$ and let $\mathcal{C}$ be the set of Hopf circles of $\partial B$ contained in $K$. Then there is a regular Hamiltonian perturbation $\left(B_{t}\right)_{t<\varepsilon}$ of $B$ with $B_{t} \cap \partial U=\mathcal{C}$ for any positive $t$.

As a corollary of Proposition 4.1, we get a non-removable intersection property for regular maximal symplectic packings. No ball of such a packing is completely disjoint from all other balls.

Corollary 4.4 (Non-removable intersection). Let $\left(B_{i}\right)_{i=1, \ldots, k}$ be a regular maximal symplectic packing of $M$. Then

$$
\text { for all } i=1, \ldots, k, \quad B_{i} \cap\left(\bigcup_{j \neq i} B_{j}\right) \neq \emptyset .
$$

Moreover, this intersection contains at least one Hopf circle of $\partial B_{i}$.

Proof. Call $\varphi_{i}$ the symplectic embeddings of $B\left(r_{i}\right)$ into $M$ corresponding to $B_{i}$. Suppose by contradiction that $K:=B_{i} \cap\left(\bigcup_{j \neq i} B_{j}\right)$ contains no Hopf circle of $\partial B_{i}$. The map $\varphi_{i}$ is a regular symplectic embedding of $\overline{B\left(r_{i}\right)} \backslash K$ into the open symplectic manifold $M^{\prime}=M \backslash \bigcup_{j \neq i} B_{j}$. By Proposition 4.1, $\varphi_{i}$ can be 'extended' to a symplectic embedding $\widetilde{\varphi}_{i}: B\left(r_{i}+\varepsilon\right) \hookrightarrow M^{\prime}$. The maps $\varphi_{1}, \ldots, \widetilde{\varphi}_{i}, \ldots, \varphi_{k}$ thus provide a symplectic packing of $M$ by $k$ balls of radii $\left(r_{1}, \ldots, r_{i}+\varepsilon, \ldots, r_{k}\right)$. This contradicts the definition of a maximal symplectic packing. 


\section{Non-smooth symplectic packings}

We turn to the problem of identifying non-smooth (but regular) maximal symplectic packings. We first prove the existence of supporting surfaces of such packings (Theorem 3 ). We then remark that passing from Theorem 3 to the precise statement of Theorem 4 is a matter of being able to perturb the topological supporting surface to a smooth symplectic surface (Lemma 5.1). We finally show that the smoothening is possible under the hypothesis of Theorem 4, thus proving it.

\subsection{Existence of a supporting surface: proof of Theorem 3}

The idea of the proof is very simple. The non-removable intersection property of the packing implies the existence of a Hopf circle $C_{1}$ of $\partial B_{1}$ contained in the union of the other balls. Let us denote by $\mathcal{C}_{1 i}$ the set of circles of $B_{i}$ intersecting $C_{1}$ along an open set $\left(\mathcal{C}_{1 i}\right.$ may be empty or contain several circles). Denote by $S_{0}$ the Hopf disc in $B_{1}$ bounded by $C_{1}$ and by $S_{1}$ the surface obtained by gluing the Hopf discs corresponding to $\mathcal{C}_{1 i}$ to $S_{0}$. Obviously no point of $C_{1}$ is a boundary point of $S_{1}$. Now there are two possibilities. Either the $\mathcal{C}_{1 i}$ are covered by the balls $B_{j}$ for $j \neq i$ or not. In the first case, we can glue to $S_{1}$ the Hopf discs corresponding to the circles of $\mathcal{C}_{1 i j}\left(\mathcal{C}_{1 i j}\right.$ is the set of circles of $\partial B_{j}$ intersecting a circle of $\mathcal{C}_{1 i}$ in an open set). We obtain a surface $S_{2}$ with boundary points neither in $C_{1}$ nor in $C_{1 i}$, which we can use in order to iterate the construction. In the latter case, we can get rid of one of the circles of $C_{1 i}$ from the intersections between the balls by a small Hamiltonian perturbation. The effect of this transformation is that $C_{1}$ is not covered by the other balls any more. It actually means that $C_{1}$ was not relevant for our purpose, but there clearly exists another circle of $\partial B_{1}$ to which we can apply the previous procedure. The reason why this iteration process stops and produces a closed surface is the finiteness condition on the singularities. In order to make the preceding iterative process rigorous and clear, we encode the situation into a graph.

Let $B_{1}, \ldots, B_{k}$ be the balls of a regular maximal symplectic packing of $M$. We already know how to associate a supporting topological sphere to any common Hopf circle of two balls of the packing (see Lemma 3.4). We thus consider in the following only those Hopf circles not concerned by this basic construction. Define

$$
\begin{aligned}
& \mathcal{S}_{i}:=\left\{x \in \partial B_{i} \mid \exists j \neq i, C_{x} \subset \partial B_{j}\right\}, \\
& \mathcal{C}_{i}:=\left\{x \in \partial B_{i} \mid C_{x} \subset \bigcup_{j \neq i} \partial B_{j}, C_{x} \not \subset \partial B_{j} \forall j \neq i\right\},
\end{aligned}
$$

where $C_{x}$ denotes the possibly singular Hopf circle of $\partial B_{i}$ passing through $x$. Clearly, if one of the $\mathcal{C}_{i}$ is empty, then Corollaries 4.4 and 3.4 show that there must be a supporting 2-sphere of the packing passing through $B_{i}$. As before, our aim is to explain to what $\mathcal{C}_{i}$ can be reduced after Hamiltonian perturbation of the packing. Notice that, by definition, each circle of $\mathcal{C}_{i}$ contains at least one triple intersection point between the balls of the packing, and there are only finitely many such points because of the regularity condition on the packing. Since any two Hopf circles of a given ball are disjoint, each $\mathcal{C}_{i}$ thus contains only a finite number of Hopf circles of $\partial B_{i}$. Consider henceforth the finite graph $G$ whose vertices are the Hopf circles contained in one of the $\mathcal{C}_{i}$, and the edges are the pairs of such circles which share an open arc. Also colour the vertices black when they represent a circle $C \in \mathcal{C}_{i}$ which is also contained in $\bigcup_{j \neq i} \mathcal{C}_{j}$ and red otherwise.

A red vertex is a Hopf circle $C$ of $\partial B_{i}$ which is covered by the other balls but not by the union of the $\mathcal{C}_{j}$ for $j \neq i$. Since each $\mathcal{C}_{j}$ is compact, there must be an open $\operatorname{arc} I \subset C$ which is a piece of a Hopf circle of $\partial B_{j}$, not covered itself by the other balls of the packing. The perturbation procedure then allows us to produce a new packing $\left(\widetilde{B}_{1}, \ldots, \widetilde{B}_{k}\right)$ very close to the original one, with intersection between the balls unchanged except that $\widetilde{B}_{i} \cap \widetilde{B}_{j}=B_{i} \cap B_{j} \backslash I$. The graph $\widetilde{G}$ associated to the perturbed maximal packing is thus a subgraph of $G$ obtained by erasing the vertex $C$ together with 


\section{E. Opshtein}

all its adjacent edges, and turning all its neighbours in $G$ to red. In particular, $\widetilde{G}$ has one vertex less than $G$. This process can be iterated as long as there is a red vertex in the graph. Because the initial graph is finite, there must be a stabilization after a finite number of steps. We conclude that some Hamiltonian perturbation of the packing leads to a graph which is only black. We will now suppose that $G$ itself has only black vertices. Applying the perturbation procedure once more, we can arrange that

$$
B_{i} \cap \bigcup_{j \neq i} B_{j}=\mathcal{C}_{i} \cup \mathcal{S}_{i}
$$

We claim that each connected component $\hat{G}$ of $G$ corresponds to a supporting surface. Actually, let $S$ be the union of the Hopf discs corresponding to the Hopf circles of $\hat{G}$. It is obviously a connected space, covered by the closed balls of the packing. We need however a brief discussion of the regularity of this space before we can assure that it is a topological surface. Inside the balls, $S$ is an immersed symplectic surface, whose only self-intersection points are positive and located at the origin of the balls. Consider now a point $x$ of $S \cap \partial B_{i}$ which is not a singular point of any ball. In particular it is not a triple intersection point of the packing, so $S$ cannot be made of more than two discs in a neighbourhood. Moreover $x$ belongs to a Hopf circle of $\mathcal{C}_{i}$. The fact that this Hopf circle is black coloured implies that $x$ is in the boundary of at least two Hopf discs. It follows that $S$ is locally made of exactly two smooth Hopf discs glued along an interval around $x$. It is easily checked that $S$ is even locally diffeomorphic to a cylinder $\{y=|x|\} \times \mathbb{R}$ at these points. We thus conclude that $S$ is indeed a closed topological surface with finitely many possible singular points located at the singular points of the packing.

\subsection{Refinement of Theorem 3 when the supporting surfaces are smooth}

In the concrete case of five balls in $\mathbb{P}^{2}$, we explain now how to sharpen Theorem 3 and get Theorem 4 . We consider for the remainder of this paragraph a regular maximal symplectic packing of $\mathbb{P}^{2}$ by five equal balls $\varphi_{1}, \ldots, \varphi_{5}$ (or $B_{1}, \ldots, B_{5}$ ), of radii $\sqrt{2 / 5}$, and a supporting surface $S$ of the packing. Then $S$ is made of several Hopf discs, each of which is of area $2 / 5 \pi$ because its boundary is a Hopf circle of the boundary of a ball. Since the Fubini-Study form is integral and $S$ is a closed surface, the number of discs is a multiple $5 k$ of 5 , the symplectic area of $S$ is $2 k \pi$, and its homology class is $2 k[L]\left(L\right.$ is a line in $\left.\mathbb{P}^{2}\right)$.

Lemma 5.1. Assume that there is a smooth symplectic immersion $\mathcal{C}^{0}$-close to $S$, whose only selfintersections are at the origins of the balls. Then $S$ is of area $2 \pi$, made of one Hopf disc in each ball of the packing, and it is the unique supporting surface of the packing.

Proof. Denote by $k_{i}$ the number of Hopf discs of $S \cap B_{i}$, so that $k_{1}+k_{2}+\cdots+k_{5}=5 k$. The self-intersection of $S$ near the origin $O_{i}$ of $B_{i}$ (namely the number of double points of generic perturbations of $S$ near $O_{i}$ ) is then given by

$$
\delta_{i}=\frac{k_{i}\left(k_{i}-1\right)}{2} .
$$

The assumption on the smoothening of $S$ means that one can find a symplectically immersed surface $\widetilde{S}$ homologous to $S$ (hence in the homology class $2 k[L]$ ) with positive self-intersections. They are located in small neighbourhoods of the $O_{i}$ and are the same as those of $S \cap B_{i}$. The total selfintersection number of $\widetilde{S}$ is thus

$$
\delta:=\sum_{i=1}^{5} \delta_{i}=\sum_{i=1}^{5} \frac{k_{i}\left(k_{i}-1\right)}{2} .
$$


MaXimal SYMPLECTIC PACKINGS IN $\mathbb{P}^{2}$

Taking into account that $\sum k_{i}=5 k$, we easily get that

$$
\delta \geqslant \frac{5 k(k-1)}{2} .
$$

The positivity of the self-intersections together with the fact that $\widetilde{S}$ is symplectic imply that $\widetilde{S}$ is actually a $J$-holomorphic curve for an almost complex structure on $\mathbb{P}^{2}$ (see [AL94]). It must therefore satisfy the adjunction inequality, which gives in our present situation [MS98]:

$$
\delta \leqslant \frac{(2 k-1)(2 k-2)}{2}=(2 k-1)(k-1) .
$$

It follows from (3) and (4) that $k=1$ and $\delta=0$. We thus conclude that $S$ was made of five Hopf discs, one in each ball. Finally, we argue by contradiction to prove that $S$ is the only supporting surface of the packing. Assume that there is a supporting surface $S^{\prime}$ distinct from $S$, made of $k_{i}^{\prime}$ Hopf discs in each ball, and of total symplectic area $2 k^{\prime} \pi$ (so that $\sum k_{i}=5 k^{\prime}$ ). Since the only intersection points between $S$ and $S^{\prime}$ are the centres of the balls, we get

$$
4 k^{\prime}=2 L \cdot 2 k^{\prime} L=S \cdot S^{\prime}=\sum_{i=1}^{5} S \cdot O_{i} S^{\prime}=\sum_{i=1}^{5} k_{i} \cdot 1=5 k^{\prime} .
$$

This is the desired contradiction.

Note that the previous computation can be made for seven (respectively eight) balls. When they are symplectically smoothable in the previous sense, the supporting surfaces are at most seven (respectively eight), all of area $3 \pi$ (respectively $6 \pi$ ). Each one intersects six of the balls through one Hopf disc and the last one through two Hopf discs (respectively intersects seven of the balls through two Hopf discs and the last one through three).

\subsection{Smoothening of the supporting surfaces for packings of simple type}

In this paragraph, we show that the smoothening required by Lemma 5.1 can be achieved when precise conditions on the singularities of the packings are given. Recall that the singularities of $S$ are the union of a finite set of singularities of the packing, segments of the characteristic foliations joining precisely these points, and more exceptionally full Hopf circles. The first step is to deal with the generic singular points of $S$.

Lemma 5.2. Let $S$ be a symplectic surface singular along a segment or a circle $\Gamma$. Assume that a neighbourhood of $\Gamma$ in $S$ is

(i) either globally diffeomorphic to

$$
\left.\{y=\alpha(z)|x|\} \subset \mathbb{R}^{2}(x, y) \times\right] 0,1[(z),
$$

where $\alpha$ is a continuous function which vanishes at 0 and 1 if $\Gamma$ is a segment,

(ii) or locally diffeomorphic to $\{y=\alpha(z)|x|\}$, where $\alpha$ is continuous if $\Gamma$ is a circle. Then $S$ can be smoothened to a symplectic surface by a $\mathcal{C}^{0}$-perturbation.

Proof. Consider first the case of a segment $\Gamma$ of singularities. Using Moser's argument, a neighbourhood of $\Gamma$ can be presented symplectically as the cylinder

$$
V_{\varepsilon}:=\left\{\left|x_{1}\right|<1+\varepsilon,\left|y_{1}\right|<\varepsilon,\left|z_{2}\right|<\varepsilon\right\} \subset \mathbb{C}^{2}\left(z_{1}=x_{1}+i y_{1}, z_{2}\right)
$$

in such a way that $\Gamma$ corresponds to $[-1,1] \times\{0\}$ and $S$ corresponds to a union of two symplectic surfaces $S_{1}, S_{2}$ with common boundary $\Gamma$. Since $S_{1}$ and $S_{2}$ are symplectic, the identification can be done so that also $T_{q} S_{1}=\operatorname{Span}_{\mathbb{R}}\left(\partial / \partial x_{1}, \partial / \partial y_{1}\right)$ and $T_{q} S_{2}=\operatorname{Span}_{\mathbb{R}}\left(\partial / \partial x_{1},-\partial / \partial y_{1}-u\left(x_{1}\right) \partial / \partial z_{2}\right)$ $(q \in \Gamma)$ where $u\left(x_{1}\right)$ is a continuous complex-valued function along $\Gamma$ which vanishes for $\left.x_{1} \notin\right]-1,1[$. 


\section{E. Opshtein}

If the neighbourhood of $\Gamma$ is small enough, $S_{1}$ can be straightened to the strip $A=\left\{\left|x_{1}\right|<1+\varepsilon\right.$, $\left.0 \leqslant y_{1}<\varepsilon, z_{2}=0\right\}$ by a map $h$ which is $\mathcal{C}^{1}$-close to the identity (and even tangent to the identity along $\Gamma$ ). This map may distort $\omega$ but by no more than an $\varepsilon$-factor. We produce our symplectic smoothening of $S$ by cutting $S_{1}$ and replacing it by a 'very symplectic' surface $\Sigma_{u} \subset V_{\varepsilon}$ which interpolates smoothly between $\left(\Gamma, \operatorname{Span}_{\mathbb{R}}\left(\partial / \partial x_{1}, \partial / \partial y_{1}+u\left(x_{1}\right) \partial / \partial z_{2}\right)\right)$ and $A$.

To this purpose, we consider a smooth 'profile' of maps $\varphi_{v}:[0, \varepsilon[\longrightarrow \mathbb{C}$ parameterized by $\mathbb{C}$, such that $\varphi_{v}(0)=0, \dot{\varphi}_{v}(0)=v, \varphi_{v} \equiv 0$ on $\left[\varepsilon / 2, \varepsilon\left[\right.\right.$ and $\varphi_{0} \equiv 0$. Up to shrinking these maps, we can also arrange that $\partial \varphi_{v} / \partial v$ and $\dot{\varphi}_{v}$ are very small on big compact sets (for $v$ ). Then the surface

$$
\Sigma_{u}:=\left\{\left(x_{1}, y_{1}, \varphi_{u\left(x_{1}\right)}\left(y_{1}\right)\right),\left|x_{1}\right|<1+\varepsilon, 0 \leqslant y_{1}<\varepsilon\right\} \subset \mathbb{C}^{2}
$$

obviously interpolates $\left(\mathcal{C}^{1}\right.$-) smoothly between $\left(\Gamma, \partial / \partial y_{1}+u\left(x_{1}\right) \partial / \partial z_{2}\right)$ and $A$. Observe moreover that the tangent vectors to $\Sigma_{u}$,

$$
v_{1}:=\frac{\partial}{\partial x_{1}}+\left(\frac{\partial \varphi_{v}}{\partial v} \cdot \frac{\partial u}{\partial x_{1}}\right) \frac{\partial}{\partial z_{2}} \quad \text { and } \quad v_{2}:=\frac{\partial}{\partial y_{1}}+\left(\frac{\partial \varphi_{v}}{\partial y_{1}}\right) \frac{\partial}{\partial z_{2}},
$$

are small perturbations of $\partial / \partial x_{1}$ and $\partial / \partial y_{1}$ respectively provided that $\varphi$ is sufficiently small in the $\mathcal{C}^{1}$-norm. The tangent planes to $\Sigma_{u}$ are thus far from being Lagrangian.

If $\Gamma$ is now a circle of singularities, consider a subsegment $\Gamma_{1} \subset \Gamma$. As above, a neighbourhood of $\Gamma_{1}$ in $S$ is the union of two smooth strips $S_{1}$ and $S_{2}$ glued along $\Gamma_{1}=[-1,1] \times\{0\} \subset \mathbb{C}^{2}$. Their tangent planes along $\Gamma$ are $T_{q} S_{1}=\operatorname{Span}_{\mathbb{R}}\left(\partial / \partial x_{1}, \partial / \partial y_{1}\right)$ and $T_{q} S_{2}=\operatorname{Span}_{\mathbb{R}}\left(\partial / \partial x_{1},-\partial / \partial y_{1}-u\left(x_{1}\right) \partial / \partial z_{2}\right)$, where $u\left(x_{1}\right)$ is a continuous complex-valued function. The only difference with the previous situation is that $u$ may not vanish at the boundary of $\Gamma_{1}$. Consider any smooth function $v:[-1,1] \longrightarrow \mathbb{C}$ vanishing at -1 and 1 , which coincides with $u$ on $\Gamma_{2}=[-1 / 2,1 / 2]$. Modifying $S$ exactly as above by $\Sigma_{v}$ (instead of $\Sigma_{u}$ ), we obtain a symplectic $\mathcal{C}^{0}$-perturbation $\widetilde{S}$ of $S$ which is no longer singular on the circle $\Gamma$ but on the segment $\Gamma \backslash \Gamma_{2}$. By construction, the singularity of $\widetilde{S}$ has the form (5) and a $\mathcal{C}^{0}$-perturbation of $\widetilde{S}$ is a smooth symplectic surface.

Hence all the difficulty of our desingularization problem concentrates at the (unavoidable) intersection of $S$ with singular points of the packing. To understand the situation in the greatest generality, we need local models for the singularities that may arise in regular packings. We do not pretend to find them in this paper. Instead, and rather as an illustration, we focus on the very special type of singularities which appear in the examples we constructed in $\S 2$.

DeFinition 5.3. We say that a boundary singularity $p=\varphi(q)$ of a symplectic ball $\{\varphi, B\}$ is simple if $\varphi$ is continuously differentiable at $q$, with non-vanishing derivatives in any directions but the characteristic one. A regular packing is said to be of simple type if all the singularities are simple, and if there exists no intersection point between any four balls (we only allow triple intersection points).

Before proving Theorem 4, let us discuss the meaning of this definition in our context. Consider a regular symplectic ball $\{\varphi, B\}$ of a symplectic manifold and a simple singularity $p$ of $B$. Denote by $C_{p}$ and $D_{p}$ the Hopf circle and disc passing through $p$. For $q=\varphi(\eta) \in C_{p}$, note also that $T^{c}(q):=\varphi^{\prime}(\eta) \cdot T_{\eta}^{\mathbb{C}} \partial B$ and $\pi(q):=T^{c}(q)^{\perp \omega}$. Then $D_{p}$ is tangent to $\pi(q)$ along $C_{p}$ and, from the definition of a simple singularity, both symplectic plane distributions $T^{c}(q)$ and $\pi(q)$ have a welldefined limit $T^{c}(p)$ and $\pi(p)$ when $q$ goes to $p$. In particular, $D_{p}$ has a tangent plane $\pi(p)$ at $p$. Although $T_{q} C_{p}$ may not have a limit at $p, C_{p}$ is tangent to $\pi(p)$ at $p$. Observing that the tangent plane to $\partial B$ at $q \in C_{p}$ is $T^{c}(q) \oplus T_{q} C_{p}$ we see that $\partial B \cap U$ is $\mathcal{C}^{0}$-close to the cylinder $\pi\left(C_{p}\right) \times T^{c}(p)$ (and $B \cap U$ is close to $D_{p} \times T^{c}(p)$ ). This remark immediately yields the following lemma.

Lemma 5.4. Let $B$ be a symplectic ball with a simple singularity at $p \in \partial B$. With the notation above, there is a neighbourhood $U$ of $p$ such that the linear projection $\pi: \overline{D_{p}} \cap U \longrightarrow \pi_{p}$ along $T^{c}(p)$ is an injective map. 


\section{MaXimal SYMPLECTIC PACKINGS IN $\mathbb{P}^{2}$}

This lemma prevents $D_{p}$ from spiralling too much above its tangent plane, creating problematic cone singularities. The proof of Theorem 4 is achieved in two steps. First, we smoothen the supporting surface at each (simple type) singularity of the packing. Being cautious enough in the first step allows us to use Lemma 5.2 to get rid of the remaining circles or segments of singularities.

Proof of Theorem 4. Consider a maximal symplectic packing $\left\{B_{1}, \ldots, B_{k}\right\} \subset \mathbb{P}^{2}$ of simple type, and one of its supporting surfaces $S$. Let $\left\{p_{1}, \ldots, p_{n}\right\}$ be the singularities of the packing which belong to $S$. Then the singularities of $S$ consist of the points $p_{i}$ themselves, together with open segments of the characteristic foliation linking the $p_{i}$. The differentiable model of the singularities along these segments is locally $\{y=|x|\} \times \mathbb{R} \subset \mathbb{R}^{3}$. As announced, we are going to smoothen $S$ near the $p_{i}$ in such a way that the remaining segments of singularities have the global form (5). Consider henceforth one of the singular points $p:=p_{i}$. Recall that $p$ can only be an intersection between two or three balls. Assume first that $p$ lies in the intersection between two balls only, say $B_{1}$ and $B_{2}$. Let us distinguish between two cases.

Case (a): $\pi_{1}(p)=\pi_{2}(p), T_{1}^{c}(p)=T_{2}^{c}(p)$. All indices refer to the balls of the packing (for instance, $\pi_{1}(p)$ is the plane defined above for the ball $\left.B_{1}\right)$. Consider local symplectic coordinates taking $p$ to the origin in $\mathbb{C}^{2}$ and $\pi(p)$ to $\left\{z_{2}=0\right\}$. Inside a small bidisc $Q_{\varepsilon}:=\left\{\left|z_{1}\right|<\varepsilon\right\} \times\left\{\left|z_{2}\right|<\varepsilon\right\}$, the surface $S$ together with its tangent planes are very close to $\left\{z_{2}=0\right\}$. The projection $\pi: S \cap Q_{\varepsilon} \longrightarrow\left\{z_{2}=0\right\}$ is therefore a covering map, which must be injective by Lemma 5.4. The intersection of $S$ with $\partial Q_{\varepsilon}$ is therefore the graph over $\partial \mathbb{D}_{\varepsilon}$ of a piecewise $\mathcal{C}^{1}$-smooth complex-valued function with small $\mathcal{C}^{1}$-norm. This function can obviously be extended to $\mathbb{D}_{\varepsilon}$ in such a way that its graph $\Sigma \subset Q_{\varepsilon}$ is tangent to $S$ on $\partial Q_{\varepsilon}$ (except at the singularities of $S \cap \partial Q_{\varepsilon}$ ), and that its singularities are located on segments inside $\left\{\varepsilon / 2<\left|z_{1}\right|<\varepsilon\right\}$ and have the form of Lemma 5.2. Moreover, this extension can be chosen $\mathcal{C}^{1}$-small, so that $\Sigma$ remains a symplectic surface. Cutting $S \cap Q_{\varepsilon}$ and replacing it by $\Sigma$ thus gives the desired smoothening of $S$ at $p$.

Case (b): $\pi_{1}(p) \neq \pi_{2}(p)$. Consider a small neighbourhood $U$ of $p$ such that the common Hopf circle $C_{p}:=C_{p 1}=C_{p 2}$ of $\partial B_{1}, \partial B_{2}$ passing through $p$ is the union of two smooth (open) arcs $\ell_{l}$ and $\ell_{r}$ meeting at $p$. Assume also that $\pi_{1}(q) \neq \pi_{2}(q)$ for all $q \in \ell_{l} \cup \ell_{r}$. Inside $U$ we have $T_{q} C_{p}=\pi_{1}(q) \cap \pi_{2}(q)$ so that $\ell_{l}$ and $\ell_{r}$ have common tangency $\pi_{1}(p) \cap \pi_{2}(p)$ at $p$. Since $D_{i}(p)$ has limit tangent plane $\pi_{i}(p)$ at $p$, if $\ell_{l} \cup \ell_{r}$ is $\mathcal{C}^{1}$-smooth then the model of the singularity of $S$ at $p$ is the same as at any generic point of $C_{p}$, and we can forget it. Otherwise $\ell_{l} \cup \ell_{r}$ is a $\mathcal{C}^{1}$ cusp, meaning that its projection to $\pi_{1}(p) \cap \pi_{2}(p)$ is a half-line (see Figure 3 ). Then $C_{p} \times T_{1}^{c}(p)$ $\left(=C_{p} \times T_{2}^{c}(p)\right)$ separates $U$ into two cylinders, a big one (with aperture $2 \pi$ at $\left.p\right)$ and a small one, in the neighbourhood of which each ball is contained. To fix the ideas, suppose that $B_{1}$ is the 'big ball' in $U$, and consider symplectic coordinates in $U$ such that $p=0, \pi_{1}(p)=\left\{z_{2}=x_{2}+i y_{2}=0\right\}$ and $\pi_{1}(p) \cap \pi_{2}(p)=\operatorname{Span}_{\mathbb{R}}\left(\partial / \partial y_{1}\right)$. Note that $\pi_{2}(p)$ is transverse to $\left\{z_{1}=0\right\}$ because it is symplectic and contains $\partial / \partial y_{2}$. So the projection of $S$ on $\pi_{1}(p)$ along $T_{1}^{c}(p)$ inside a small bidisc $Q_{\varepsilon}$ is a covering map, injective by Lemma 5.4. The intersection $\partial Q_{\varepsilon} \cap S$ is henceforth the graph of a piecewise $\mathcal{C}^{1}$ smooth complex-valued function over $\partial \mathbb{D}_{\varepsilon}$. It is easy to see that this map has bounded derivatives and small $\mathcal{C}^{0}$-norm. The same procedure as in case (a) above produces the smoothening.

Assume now that $p$ lies in the boundary of three balls $B_{1}, B_{2}, B_{3}$. In a sufficiently small neighbourhood $U$ of $p$, each intersection $B_{i} \cap B_{j} \cap U$ is a smooth open arc $\ell_{i j}$ ending at $p$. Reasoning as in cases (a) and (b), it is easy to smoothen $S$ at $p$ when $\pi_{1}(p)=\pi_{2}(p)=\pi_{3}(p)$ or $\pi_{1}(p)=\pi_{2}(p) \neq \pi_{3}(p)$. Thus it only remains to investigate the situation of three different tangent planes for $D_{i}(p)$ at $p$ (see Figure 4). The curves $\ell_{i j}$ then have well-defined tangencies at $p$ :

$$
T_{p} \ell_{i j}=\pi_{i}(p) \cap \pi_{j}(p) .
$$

Consider a parameterization of the $\ell_{i j}$ by smooth maps $\gamma_{i j}:\left[0, \varepsilon\left[\longrightarrow U \subset \mathbb{C}^{2}\right.\right.$ with $\gamma_{i j}(0)=p$. Notice that $\omega\left(\dot{\gamma}_{i j}(0), \dot{\gamma}_{j k}(0)\right)>0$ because $\pi_{j}(p)=\operatorname{Span}_{\mathbb{R}}\left(\dot{\gamma}_{i j}(0), \dot{\gamma}_{j k}(0)\right)$ is a symplectic plane. Applying a 


\section{E. Opshtein}

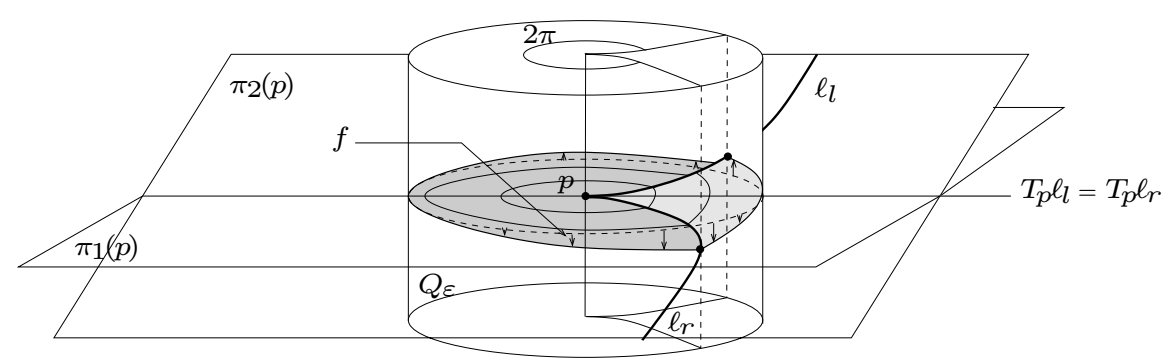

Figure 3. Singularity of type $\pi_{1}(p) \neq \pi_{2}(p)$ and $C_{p}$ is not smooth.

symplectic linear change of coordinates and a rescaling of the parameterizations, we can suppose that

$$
\dot{\gamma}_{12}(0)=\left(\begin{array}{l}
1 \\
0 \\
1 \\
0
\end{array}\right), \quad \dot{\gamma}_{23}(0)=\left(\begin{array}{l}
0 \\
1 \\
1 \\
0
\end{array}\right), \quad \dot{\gamma}_{31}(0)=\left(\begin{array}{r}
-1 \\
-1 \\
1 \\
0
\end{array}\right) \subset \mathbb{C}^{2} \approx \mathbb{R}^{4} .
$$

Looking sufficiently close to $p$, there is a diffeomorphism $\Phi$ that is $\mathcal{C}^{1}$-close to the identity taking $S$ to the cone over the triangle:

$$
\Sigma:=\left\{\left(\begin{array}{c}
z \varphi_{1}(\theta) \\
z \\
0
\end{array}\right), 0<h<1\right\},
$$

where $\varphi_{1}(\theta)$ is the counterclockwise parameterization of the triangle $T_{1}$ spanned by the points $(1,0)$, $(0,1),(-1,-1)$ of $\mathbb{R}^{2}$. Consider now the polar parameterizations $\varphi_{\rho}$ of a family of convex curves $T_{\rho}$ with $T_{\rho}=\left\{x^{2}+y^{2}=\rho^{4}\right\}$ for $\rho \ll 1, T_{\rho}=\rho \cdot T_{1}$ for $\rho \simeq 1$ and the angles of the singularities of $T_{\rho}$ vary smoothly with $\rho$ (see Figure 4 ). Then the surface

$$
\widetilde{\sigma}:=\left\{\left(\varphi_{h}(\theta), h, 0\right)\right\}
$$

is smooth near the origin, has tangent planes far from being Lagrangian and coincides with $\Sigma$ in a neighbourhood of $h=\left\{y_{2}=1\right\}$. Cutting $\Sigma$ from $S$ and replacing it by $\Phi^{-1}(\widetilde{\Sigma})$, we get a symplectic smoothening of $S$ at $p$. We were also sufficiently cautious in the extrapolation from $T_{1}$ to $T_{\rho}, \rho \simeq 0$, to ensure that the remaining singularities have the form (5).

After a small perturbation of $S$, we thus obtain a symplectic surface with singularities along circles or segments, of the type required to apply Lemma 5.2. The surface $S$ can therefore be perturbed to a smooth symplectic surface, and applying Lemma 5.1 proves Theorem 4 .

\section{ACKNOWLEDGEMENTS}

I would like to express my gratitude to P. Biran, L. Polterovich and J. Y. Welschinger for showing interest in this work, and for useful and motivating discussions. 


\section{MaXimal SYMPLECTIC PACKINGS IN $\mathbb{P}^{2}$}
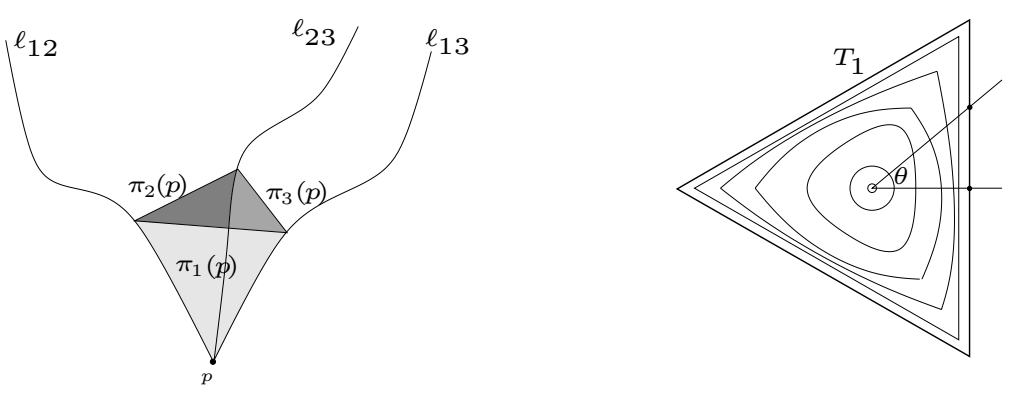

Figure 4. Singularity of $S$ and extrapolation between $T_{1}$ and 0.

\section{REFERENCES}

AL94 M. Audin and J. Lafontaine, Holomorphic curves in symplectic geometry, Progress in Mathematics, vol. 117 (Birkhäuser, Basel, 1994), 1-14.

Bir99 P. Biran, A stability property of symplectic packing, Invent. Math. 136 (1999), 123-155.

Bir01 P. Biran, Lagrangian barriers and symplectic embeddings, Geom. Funct. Anal. 11 (2001), 407-464.

Don96 S. K. Donaldson, Symplectic submanifolds and almost-complex geometry, J. Differential Geom. 44 (1996), 666-705.

Gro85 M. Gromov, Pseudoholomorphic curves in symplectic manifolds, Invent. Math. 82 (1985), 307-347.

LM95 F. Lalonde and D. McDuff, Local non-squeezing theorems and stability, Geom. Funct. Anal. 5 (1995), 364-386.

LS94 F. Laudenbach and J.-C. Sikorav, Hamiltonian disjunction and limits of Lagrangian submanifolds, Int. Math. Res. Not. (1994), no. 4, 161ff., approx. 8 pp. (electronic).

McD98 D. McDuff, From symplectic deformation to isotopy, in Topics in symplectic 4-manifolds, Irvine, CA, 1996, First International Press Lecture Series, vol. I (International Press, Cambridge, MA, 1998), 85-99.

MP94 D. McDuff and L. Polterovich, Symplectic packings and algebraic geometry, with an appendix by Y. Karshon, Invent. Math. 115 (1994), 405-434.

MS98 D. McDuff and D. Salamon, Introduction to symplectic topology, second edition, Oxford Mathematical Monographs (Clarendon Press, New York, 1998).

PPS03 G. P. Paternain, L. Polterovich and K. F. Siburg, Boundary rigidity for Lagrangian submanifolds, non-removable intersections, and Aubry-Mather theory, Mosc. Math. J. 3 (2003), 593-619, 745.

Sch05 F. Schlenk, Packing symplectic manifolds by hand, J. Symplectic Geom. 3 (2005), 313-340.

Sul76 D. Sullivan, Cycles for the dynamical study of foliated manifolds and complex manifolds, Invent. Math. 36 (1976), 225-255.

Tra95 L. Traynor, Symplectic packing constructions, J. Differential Geom. 41 (1995), 735-751.

Wei71 A. Weinstein, Symplectic manifolds and their Lagrangian submanifolds, Adv. Math. 6 (1971), 329-346.

Emmanuel Opshtein opshtein@math.u-strasbg.fr

IRMA, 7 rue René Descartes, 67084 Strasbourg cedex, France 